\title{
Phonon density of states of bulk gallium nitride
}

\author{
J. C. Nipko and C.-K. Loong ${ }^{\text {a) }}$ \\ Intense Pulsed Neutron Source Division, Argonne National Laboratory, Argonne, Illinois 60439-4814 \\ C. M. Balkas ${ }^{\text {b) }}$ and R. F. Davis \\ Department of Materials Science and Engineering, North Carolina State University, \\ Raleigh, North Carolina 27695-7907
}

(Received 25 February 1998; accepted for publication 5 May 1998)

\begin{abstract}
We report the measured phonon density of states of a bulk GaN powder by time-of-flight neutron spectroscopy. The observed one-phonon excitation spectrum consists of two broad bands centered at about 23 and $39 \mathrm{meV}$ corresponding to the acoustic and the first group of optical phonons; two sharp bands of upper optic modes at about 75 and $86 \mathrm{meV}$; and a gap of $45-65 \mathrm{meV}$. The phonon dispersion curves, lattice specific heat, and Debye temperature are calculated from fitting the data with a rigid-ion model. (C) 1998 American Institute of Physics. [S0003-6951(98)02027-0]
\end{abstract}

Recent advances in fabrication and commercialization of high-brightness blue and green light-emitting devices based on gallium nitride ${ }^{1-4}$ has renewed intense research of its basic properties. Semiconductor devices based on GaN, e.g., light-emitting diodes and diode lasers are expected to function more reliably, at higher powder and frequencies, and at elevated temperatures because of the stronger atomic bonding in the III-V nitrides as compared to the II-IV analogs. Basic thermodynamic properties such as the lattice specific heat and thermal conductivity are important design factors for the realization of current-injected laser diodes which may have to accommodate a large threshold current density and consequently high heat generation. ${ }^{2}$ Phonons are the primary excitations which influence the thermodynamic behavior. Therefore, a systematic characterization of the phonon density of states (DOS) and dispersion relations of binary nitride semiconductors is highly desirable.

Gallium nitride usually crystallizes in the hexagonal wurtzite structure at ambient pressure, although thin films having the cubic zinc-blende structure can also be grown., A variety of $\mathrm{GaN}$ single- and polycrystalline thin films have been grown on sapphire $\left(\alpha-\mathrm{Al}_{2} \mathrm{O}_{3}\right)$, spinel $\left(\mathrm{MgAl}_{2} \mathrm{O}_{4}\right), \mathrm{SiC}$, and GaAs substrates as a result of the intense efforts regarding the fabrication of $p-n$ junctions and heterostructures. These films usually show misfit and threading dislocations and other defect structures in the interfacial region due to lattice mismatch and within the bulk of the films, respectively. Numerous studies of the zone-center optic phonons in GaN films have been conducted using Raman and infrared spectroscopy, ${ }^{7-25}$ however, the question whether these data represent the bulk properties remains. Information regarding the phonon dispersion relations is not available. The growth of single crystals has been limited to rather small sizes (maximum $\mathrm{mm}$ in length) owing to the high melting point $(\sim 2800 \mathrm{~K})$ and high equilibrium pressure $(\sim 4 \mathrm{GPa})$ of nitrogen. ${ }^{26}$ Recently, an economically viable process route for producing large quantities of high-purity, single-phase

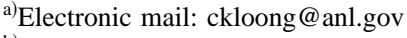

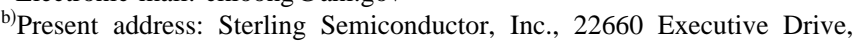
Suite 101, Sterling, VA 20166-9535.
}

GaN powders was reported by Balkas and Davis. ${ }^{27}$ Measurements of the full phonon DOS of GaN have not been reported in the literature. Neutrons can probe phonon modes throughout the Brillouin zone. The method is not restricted by selection rules and is relatively insensitive to small amount of impurities. In this letter we report the determination of the phonon DOS of GaN obtained from time-of-flight neutron spectroscopy using a bulk powder. The satisfactory interpretation of the data using a lattice-dynamic model permits the calculation of the phonon dispersion curves and the lattice specific heat.

About $50 \mathrm{~g}$ of $\mathrm{GaN}$ powder, synthesized by the reaction of liquid gallium metal with flowing ammonia and described elsewhere, ${ }^{27}$ was first characterized by conventional neutron powder diffraction at the Intense Pulsed Neutron Source (IPNS) at Argonne National Laboratory. The powder possessed the single-phase wurtzite structure, space group $P 6_{3} m c$ (at $297 \mathrm{~K}, a=3.1872(1) \AA, c=5.1818(1) \AA$, atom positions at $(1 / 3,2 / 3, u)$ and $(1 / 3,2 / 3, u+1 / 2)$ with $u=0$ for $\mathrm{Ga}$ and $u=0.3772(2)$ for $\mathrm{N}$ and the Rietveld refinement weighted $R$ factor $=9 \%$ ) which is in good agreement with the previously reported structure. ${ }^{28,29}$ The phonon DOS measurements were carried out using the high-resolution medium-energy chopper spectrometer also at IPNS. The experimental procedure and method of deriving the neutronweighted phonon DOS were given in previous reports of studies of nitride materials. ${ }^{30,31}$

The four atoms in a primitive cell of GaN give rise to 12 phonon branches. Previous Raman and IR studies focused only on six Raman-active $E_{1}, E_{2}^{(1)}, E_{2}^{(2)}$, and $2 A_{1}$ modes, and four IR-active $E_{1}$ and $2 A_{1}$ modes at the zone center. Brillouin-scattering studies were also applied to measure long-wavelength acoustic phonons. ${ }^{32-34}$ The phonon DOS, which includes contributions from all the phonons over the entire Brillouin zone, provides the essential ingredient for the calculations of various thermodynamic properties. Figure 1(a) shows the observed neutron-weighted phonon DOS of $\mathrm{GaN}$ at $8 \mathrm{~K}$. It consists of a low-energy portion $(0-45 \mathrm{meV})$, a high-energy portion $(65-95 \mathrm{meV})$, and a gap in between. ( $1 \mathrm{meV}$ is equivalent to $\left.8.066 \mathrm{~cm}^{-1}\right)$. The two broad bands at about 23 and $39 \mathrm{meV}$ contain the acoustic phonons and the 


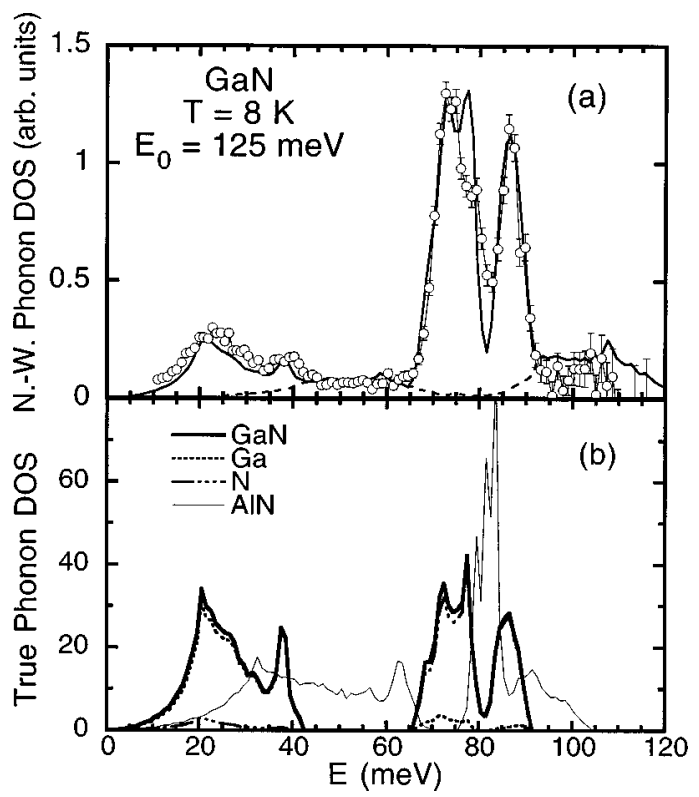

FIG. 1. (a) Measured (open circles) and calculated (lines) neutron-weighted DOS for GaN. The solid line denotes the sum of the one-phonon and twophonon DOS and the dashed line denotes the two-phonon contribution. (b) The partial and total phonon DOS of GaN calculated from the rigid-ion model. The DOS of AIN is included for comparison.

first group of optic phonons, whereas, the two sharp bands at about 75 and $86 \mathrm{meV}$ arise from the upper optic branches. The larger weight of the high-energy portion is due to the larger neutron scattering cross section of $\mathrm{N}$ (11.01 barns, 1 barn $=100 \mathrm{fm}^{2}$ ) than $\mathrm{Ga}$ (6.7 barns). The uppermost six optic modes can qualitatively be attributed to predominantly $\mathrm{N}$ vibrations, since the observed intensity is proportional to the square of vibrational amplitudes of the atoms.

A quantitative interpretation of the neutron data was realized by using a rigid-ion lattice-dynamic model. A successful application of this method to the case of AlN has been described elsewhere. ${ }^{30}$ The calculated neutron-weighted phonon DOS, shown in Fig. 1(a), reproduced all the main features of the observed spectrum as well as the zone-center mode frequencies from the optical data. Given the good agreement between the neutron results and the model, we then calculated the true partial and total DOS as well as the phonon dispersion curves along major symmetry directions in the Brillouin zone, which are shown in Figs. 1(b) and 2, respectively. Our calculated phonon DOS of GaN differs considerably from that given by Azuhata and co-workers ${ }^{7}$ who based their calculations solely on optical data. The partial DOS of $\mathrm{Ga}$ and $\mathrm{N}$ show that $93 \%$ of the $\mathrm{Ga}$ and $\mathrm{N}$

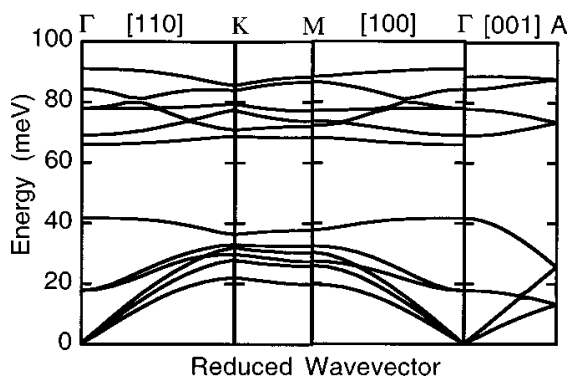

FIG. 2. The calculated phonon dispersion curves of GaN along the major symmetry directions.

Downloaded 16 Apr 2008 to 152.1.24.180. Redistribution subject to AlP license or copyright; see http://apl.aip.org/apl/copyright.jsp-

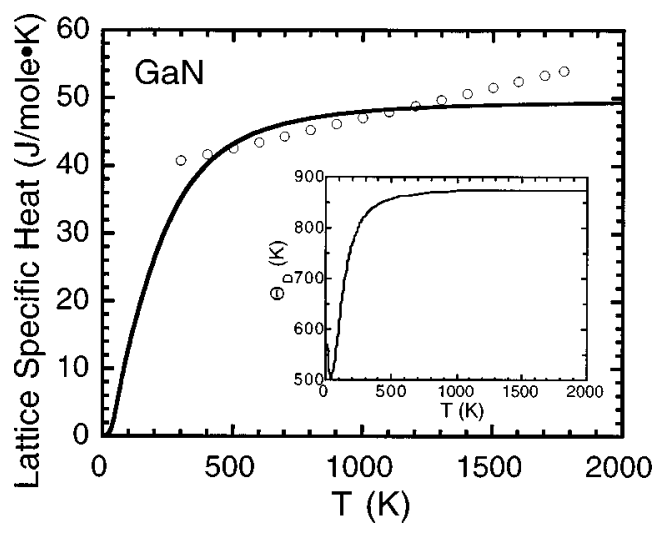

FIG. 3. The calculated (line) and measured (open circles) lattice specific heat for GaN. The inset: the calculated temperature dependence of the Debye temperature.

motion takes part in the low-energy and high-energy portion of the DOS, respectively, in agreement with a recent analysis of isotopic GaN Raman spectra. ${ }^{22}$ The positions of the zonecentered phonons, in general, do not coincide with the peaks of the DOS. This is due to the dispersion of the phonon branches across the zone-the peaks in the DOS include many unresolved critical frequencies at the zone boundaries.

The calculated lattice specific heat at constant volume, $C_{v}$, of GaN is shown in Fig. 3. To the best of our knowledge, only one set of measured specific heat values at constant pressure, $C_{p}$, has been given in the compilation by Barin. ${ }^{35}$ The reported $C_{p}$, which shows an almost linear dependence with temperature over 300-1700 K, agrees with the calculated $C_{v}$ only qualitatively. The calculated Debye temperature, $\Theta_{D}$, as a function of temperature is shown in the inset of Fig. 3. The $\Theta_{D}$ at $0 \mathrm{~K}$ is $570 \mathrm{~K}$, in excellent agreement with that $(\sim 600 \mathrm{~K})$ estimated by Slack and co-workers. ${ }^{36}$

The present results permit a comparison with the bulk properties of other binary wurtzite nitrides. In Fig. 1(b) we compare the phonon DOS of GaN and AlN. ${ }^{30}$ First, the Debye-like $\sim E^{2}$ behavior extends to a higher energy $(\sim 30$ $\mathrm{meV})$ in AlN than in $\mathrm{GaN}(\sim 20 \mathrm{meV})$. Phonons in this region are important to the low-temperature thermal conductivity. The more extensive Debye-like region of AlN implies a higher thermal conductivity of AlN at low temperatures. ${ }^{37,38}$ Second, AlN exhibits a somewhat higher one-phonon cut-off energy. The splitting of the two highenergy optic bands in $\mathrm{GaN}$ is more pronounced. This energy separation originates in part from the macroscopic electric fields generated by the net ionic charges which splits the LO-like and TO-like phonons. ${ }^{39}$ Therefore, GaN is more ionic than AlN, as also suggested by electronic band calculations. ${ }^{40}$ Third, $\Theta_{D}(0 \mathrm{~K})$ is larger for $\operatorname{AlN}(800 \mathrm{~K})$ than for GaN $(560 \mathrm{~K})$. These facts imply a higher microhardness of AlN than $\mathrm{GaN}^{41}$

Many key values of mechanical and thermal properties of bulk GaN are lacking or more data are needed over a wider temperature range. The present neutron study provides an initial comparison of the phonon and thermodynamic properties of GaN with other binary nitride semiconductors.

Work performed at Argonne National Laboratory is supported by the U.S. DOE-BES under Contract No. W-31-109- 
ENG-38. The authors are grateful to W. Reichardt for providing the GENAX computer program for the lattice-dynamic calculations.

${ }^{1}$ S. Nakamura, Solid State Commun. 102, 237 (1997).

${ }^{2}$ F. A. Ponce and D. P. Bour, Nature (London) 386, 351 (1997).

${ }^{3}$ S. Strite and H. Morkoç, J. Vac. Sci. Technol. B 10, 1237 (1992).

${ }^{4}$ R. F. Davis, K. S. Ailey, M. D. Bremser, E. Carlson, R. S. Kem, D. J. Kester, W. G. Perry, S. Tanaka, and T. W. Weeks, Jr., in Advances in Solid State Physics, edited by R. Helbig (Vieweg, Braunschweig/ Wiesbaden, 1996), Vol. 35, p. 1.

${ }^{5}$ T. D. Moustakas, T. Lei, and R. J. Molnar, Physica B 185, 36 (1993).

${ }^{6}$ H. Okumura, K. Ohta, G. Feuillet, K. Balakrishnan, S. Chichibu, H. Hamaguchi, P. Hacke, and S. Yoshida, J. Cryst. Growth 178, 113 (1997).

${ }^{7}$ T. Azuhata, T. Matsunaga, K. Shimada, K. Yoshida, T. Sota, K. Suzuki, and S. Nakamura, Physica B 219\&220, 493 (1996).

${ }^{8}$ Y. Azuhata, T. Sota, S. Suzuki, and S. Nakamura, J. Phys.: Condens. Matter 7, L129 (1995).

${ }^{9}$ R. Zhang, K. Yang, L. H. Qin, B. Shen, H. T. Shi, Y. Shi, S. L. Gu, Y. D. Zheng, Z. C. Huang, and J. C. Chen, J. Vac. Sci. Technol. A 14, 840 (1996).

${ }^{10}$ C. Wetzel, E. E. Haller, H. Amano, and I. Akasaki, Appl. Phys. Lett. 68, 2547 (1996).

${ }^{11}$ D. Kirillov, H. Lee, and J. S. J. Harris, J. Appl. Phys. 80, 4058 (1996).

${ }^{12}$ H. Sobotta, H. Neumann, R. Franzheld, and W. Seifert, Phys. Status Solidi B 174, K57 (1992).

${ }^{13}$ A. S. Barker, Jr. and M. Ilegems, Phys. Rev. B 7, 743 (1973).

${ }^{14}$ T. Kozawa, T. Kachi, H. Kano, Y. Taga, M. Hashimoto, N. Koide, and K. Manabe, J. Appl. Phys. 75, 1098 (1994).

${ }^{15}$ K. Hayashi, K. Itoh, N. Sawaki, and I. Akasaki, Solid State Commun. 77, 115 (1991).

${ }^{16}$ A. Cingolani, M. Ferrara, M. Lugará, and G. Scamarcio, Solid State Commun. 58, 823 (1986).

${ }^{17}$ P. Perlin, C. Jauberthie-Carillon, J. P. Itie, S. San Miguel, I. Grzegory, and A. Polian, Phys. Rev. B 45, 83 (1992).

${ }^{18}$ P. Perlin, A. Polian, and T. Suski, Phys. Rev. B 47, 2874 (1993).

${ }^{19}$ P. Perlin, J. Camassel, W. Knap, T. Taliercio, J. C. Chervin, T. Suski, I. Grzegory, and S. Porowski, Appl. Phys. Lett. 67, 2524 (1995).
${ }^{20}$ D. D. Manchon, Jr., A. S. Barker, Jr., P. J. Dean, and R. B. Zetterstrom, Solid State Commun. 8, 1227 (1970).

${ }^{21}$ F. Demangeot, J. Frandon, M. A. Renucci, O. Briot, B. Gil, and R. L. Aulombard, Solid State Commun. 100, 207 (1996).

${ }^{22}$ J. M. Zhang, T. Ruf, M. Cardona, O. Ambacher, M. Stutzmann, J.-M. Wagner, and F. Bechstedt, Phys. Rev. B 56, 14399 (1997).

${ }^{23}$ H. Siegle, L. Eckey, A. Hoffmann, C. Thomsen, B. K. Meyer, D. Schikora, M. Hankln, and K. Lischka, Solid State Commun. 96, 943 (1995).

${ }^{24}$ G. Wei, J. Zi, K. Zhang, and X. Xie, J. Appl. Phys. 82, 4693 (1997).

${ }^{25}$ T. Suski, P. Perlin, M. Leszczynski, H. Teisseyre, I. Gizegory, M. Bockowski, J. Jun, S. Porowski, and Z. Liliental-Weber, in III-V Nitride Materials and Processes, edited by T. D. Moustakas, J. P. Dismukes, and S. J. Pearton (The Electrochemical Society, Inc., Pennington, NJ, 1996), Vol. 96-11, p. 46.

${ }^{26}$ S. Porowski and I. Grzegory, J. Cryst. Growth 178, 174 (1997).

${ }^{27}$ C. M. Balkas and R. F. Davis, J. Am. Ceram. Soc. 79, 2309 (1996).

${ }^{28}$ H. Schulz and K. H. Thiemann, Solid State Commun. 23, 815 (1977).

${ }^{29}$ C. M. Balkas, C. Basceri, and R. F. Davis, Powder Diffr. 10, 266 (1995).

${ }^{30}$ J. C. Nipko and C.-K. Loong, Phys. Rev. B 57, 10550 (1998).

${ }^{31}$ C.-K. Loong, J. W. Richardson, Jr., S. Suzuki, and M. Ozawa, J. Am. Ceram. Soc. 79, 3250 (1996).

${ }^{32}$ Y. Takagi, M. Ahart, T. Azuhata, T. Sota, K. Suzuki, and S. Nakamura, Physica (Utrecht) 219\&220, 547 (1996).

${ }^{33}$ M. Yamaguchi, T. Yagi, T. Azuhata, T. Sota, K. Suzuki, S. Chichibu, and S. Nakamura, J. Phys.: Condens. Matter 9, 214 (1997).

${ }^{34}$ A. Polian, M. Grimsditch, and I. Grzegory, J. Appl. Phys. 79, 3343 (1996).

${ }^{35}$ I. Barin, Thermochemical Data of Pure Substances (VCH, Weinheim, 1995).

${ }^{36}$ G. A. Slack, J. Phys. Chem. Solids 34, 321 (1973).

${ }^{37}$ G. A. Slack, R. A. Tanzilli, R. O. Pohl, and J. W. Vandersande, J. Phys. Chem. Solids 48, 641 (1987).

${ }^{38}$ E. K. Sichel and J. I. Pankove, J. Phys. Chem. Solids 38, 330 (1977).

${ }^{39}$ C. A. Arguello, D. L. Rousseau, and S. P. S. Porto, Phys. Rev. 181, 1351 (1969).

${ }^{40}$ W. A. Harrison and S. Ciraci, Phys. Rev. B 10, 1516 (1974).

${ }^{41}$ M. D. Drory, Mater. Res. Soc. Symp. Proc. 468, 201 (1997). 